\title{
Towards X-ray induced transient grating methods for nanometer scale dynamics: Diffraction on transient structures induced by extreme ultraviolet radiation from FLASH
}

\author{
A. Föhlisch ${ }^{1, a}$, M. Beye ${ }^{1}$, H. Redlin ${ }^{2}$, and S. Düsterer ${ }^{2}$ \\ 1 Institute for experimental physics, Hamburg University, Luruper Chaussee 149, 22761 Hamburg, \\ Germany \\ 2 Hamburger Synchrotron Strahlungslabor at Deutsche Elektronen Synchrotron HASYLAB/DESY, \\ Notkestr. 85, 22607 Hamburg, Germany
}

\begin{abstract}
Using the high brilliance femtosecond soft X-ray pulses from the FreeElectron LASer at Hamburg (FLASH) the X-ray induced transient optical reflectivity change of GaAs has been established as a versatile method for femtosecond $\mathrm{X}$-ray/optical cross-correlation [1]. As the underlying physical mechanism is the $\mathrm{X}$-ray induced dynamics within solids, we present in this work a feasibility study how transient grating methods could be used to study nanometer scale dynamics in materials, such as the radical diffusion parameters in photoresist materials for EUV lithography.
\end{abstract}

\section{Introduction}

X-ray free-electron lasers (FEL) provide the unique opportunity to address the intersecting point of many modern scientific disciplines - namely to disentangle the function and the temporal evolution of complex systems, where only few active sites and atoms contribute in a matrix of many others, as it is the case for most of the materials science, chemistry and biology we rely on. In addition, the response of matter to extreme electromagnetic fields leads both to fundamental physics and insights into regions far from application such as interstellar chemistry. The advantage of the X-ray spectral region is that selected atomic centers can be excited and probed separately, since X-rays interact with atomically localized element specific inner shell electrons. X-ray photon-in/photon-out spectroscopic methods take full advantage of the novel FEL radiation sources and will - fully developed - provide highly relevant tools for scientific and technological investigations as we can access matter in all aggregate states and in extreme conditions (pressure, temperature, external fields).

The power of time resolved pump-probe spectroscopy has been demonstrated with optical lasers and with high harmonic pulses in the vacuum ultra violet, revolutionizing the field by unprecedented pulse definition on the attosecond timescale. However, only SASE free-electron lasers, like the free-electron laser at Hamburg [8], the XFEL in Hamburg, the Spring8 Compact SASE Source (SCSS) and the Linac Coherent Light Source (LCLS) will provide sufficiently high brilliance, intensity and coherence in the X-ray spectral range, necessary to reach the core levels of the elements relevant for chemistry, biology and materials science and investigate ultrafast dynamics on the atomic scale. We thus have to develop the full range of X-ray methods, like

\footnotetext{
a e-mail: alexander.foehlisch@desy.de
} 
resonant inelastic X-ray scattering, electron spectroscopy for chemical analysis, X-ray diffraction and coherent imaging into femtosecond time resolved methods.

Equally important to ultrafast dynamics on the atomic level is the collective dynamics on a nanometer scale within matter. In chemistry, radical and excited species diffusion are crucial processes, currently studied at optical wavelengths with laser based transient grating spectroscopy [3-6]. In physics fundamental questions are under investigation regarding phase transitions, critical fluctuations in magnetic materials as well as nanometer scale ordering phenomena regarding charge, spin and orbital order in correlated materials. In order to probe these types of nanometer scale dynamics, we should extend transient grating methods towards shorter wavelengths, using the coherent and ultrafast X-ray pulses, which FELs can deliver.

The first step to develop X-ray spectroscopic methods at free-electron lasers in combination with synchronized optical lasers towards femtosecond time resolved pump-probe methods is to find efficient ways to cross-correlate the femtosecond X-ray and optical pulses to monitor at the interaction point within the experimental set-up spatial and temporal overlap and temporal jitter. This necessity arises in particular, as beam transport from the synchronized FEL and optical Laser sources to the experimental interaction point spans between $50-100 \mathrm{~m}$. In the optical regime non-linear processes like sum frequency generation are routinely used. These processes have in the soft X-ray and X-ray region low cross sections. The alternative approach to cross-correlate optical and X-ray pulses has been side band generation in the photoemission process, which requires an ultra high vacuum environment and dedicated photoelectron analyzers [9-11]. To overcome these difficulties, we have thus developed with X-ray induced transient optical reflectivity changes a novel method of X-ray/optical cross-correlation $[1,2]$, which is highly versatile and can be applied as an all-optical method in virtually all experimental environments and for present and future FELs. Using X-ray induced changes in optical reflectivity we show in this contribution the potential to perform a transient grating experiment with X-rays in order to probe collective dynamics on the nanometer scale. With EUV radiation at $13.5 \mathrm{~nm}$ we created an X-ray induced transient grating in GaAs, which we then probed with optical diffraction.

\section{$2 \mathrm{X}$-ray induced transient optical reflectivity for X-ray/optical cross-correlation}

The principle of X-ray induced transient optical reflectivity as a X-ray/optical cross-correlator is described in Ref. [1] and the change in optical reflectivity $\Delta \mathrm{R} / \mathrm{R}$ of a GaAs surface after X-ray excitation is determined as a function of temporal delay between the X-ray pump and optical probe pulse. As shown schematically in Fig. 1, femtosecond extreme ultraviolet radiation pulses from FLASH $(39.5 \pm 0.5 \mathrm{eV}$ with $\approx 50 \mathrm{fs}$ pulse duration) impinge onto a GaAs(100) crystal at $41.5^{\circ}$ incidence angle with the electric field vector in the surface plane. With X-ray fluences less than $16 \mathrm{~mJ} / \mathrm{cm}^{2}$ we stay below the optical damage threshold energy of GaAs $[1,12]$. With delayed optical pulses of either $800 \mathrm{~nm}$ or $400 \mathrm{~nm}$ and a duration of 120-150 fs (FWHM) we probed at $53^{\circ}$ incidence the temporal response of the optical reflectivity. The reflectivity changes were detected by two fast photodiodes, monitoring the optical pulse energies prior and after reflection. In addition, we operated the optical laser at twice the FEL repetition rate in order to obtain for each X-ray pulse pumped a direct comparison to the reflectivity of the un-pumped GaAs surface. We have investigated the time constants and the physical mechanism of X-ray induced transient optical reflectivity in detail [1]. Depending on the excitation energy and the wavelength of the optical probe we monitor on the femtosecond and fast picosecond timescale the initial disturbance and relaxation of the electronic system, which couples on a slow picosecond timescale to the crystal lattice (see Fig. 2). We monitor this as marked changes to the dielectric response as seen in the rapid decrease and increase of the optical reflectivity. In particular the fast drop of reflectivity at $\tau_{X C}=160 \pm 44 \mathrm{fs}$, depicted in the inset data plot of Fig. 2, allows us to apply X-ray pulse induced transient optical reflectivity change as a tool for fs cross-correlation between the X-ray and optical laser pulses at the interaction point of the experimental set-up. The physical origin of the ultra fast change of $\Delta \mathrm{R} / \mathrm{R}$ is the absorption 


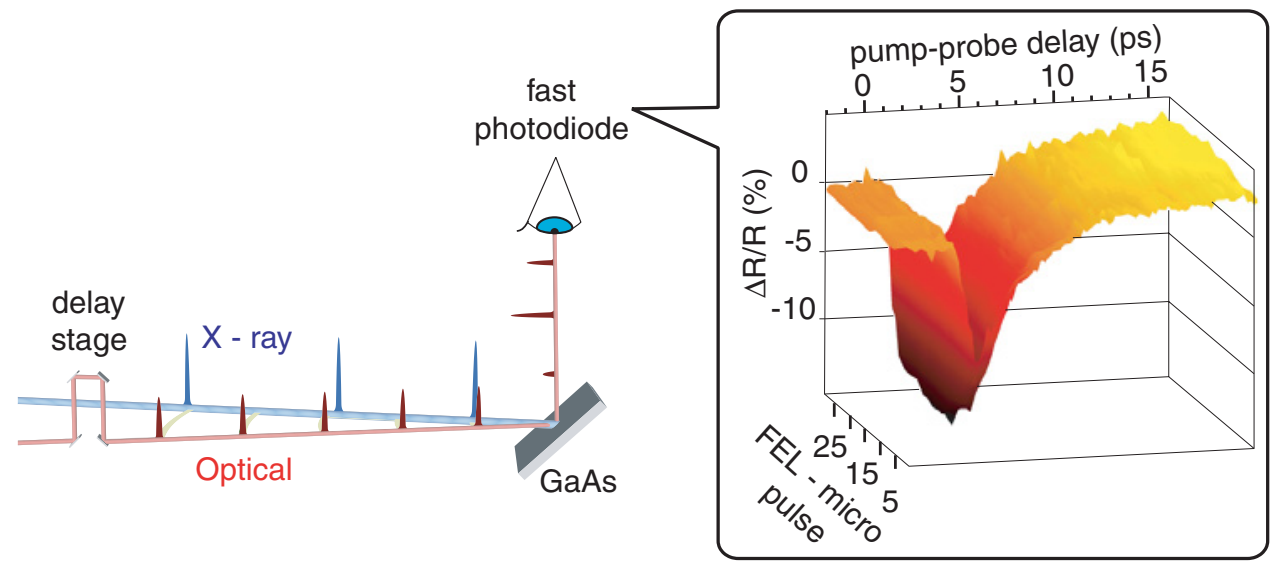

Fig. 1. A femtosecond X-ray/optical cross-correlator - experimental scheme (Ref. [1]): Transient X-ray induced optical reflectivity $(\Delta \mathrm{R} / \mathrm{R})$ is measured as a function of the temporal delay between extreme ultraviolet pump pulses $(39.5 \mathrm{eV},<50 \mathrm{fs},<16 \mu \mathrm{J})$ from the Free-Electron LASer at Hamburg (FLASH) and visible laser probe pulses $(800 \mathrm{~nm}$ or $400 \mathrm{~nm}, 120 \mathrm{fs},<10 \mathrm{~nJ})$ reflected from a crystalline GaAs $(100)$ surface. The visible laser operates at twice the repetition rate of FLASH to measure the pumped and unpumped surface as a reference.

of the FEL radiation leading preferentially to Ga 3d vacancies (atomic photoionization cross section for Ga 3d: 3.6 Mbarn [14]) which decay on the timescale of a few femtoseconds via Auger processes and autoionization into valence excitations further thermalizing by electron-electron scattering. The magnitude of the initial drop scales first linearly and later saturated with X-ray fluence [1]. After this initial ultra fast electron dynamics, electronic relaxation and electron phonon coupling leads to picosecond $\Delta \mathrm{R} / \mathrm{R}$ dynamics, that depends in detail on the optical probe wavelength. Here both the initial X-ray induced distortion of the valence electronic structure through direct photoionization and ultra fast Auger decay of inner shell vacancies, electronic screening and electron-electron scattering as well as the structural changes to the crystal lattice need to be considered [1]. A first application of our finding was the single shot X-ray optical cross-correlation measurement in a time-to-space mapping geometry to determine the arrival time of individual X-ray and optical femtosecond pulses [2].

\section{Probing dynamics on the nanometer scale: Towards $X$-ray induced transient grating methods}

Next to dynamics on the atomic scale in matter, we are interested in the collective dynamics on a nanometer scale within matter. In chemistry, radical and excited species diffusion are crucial processes, currently studied at optical wavelengths with laser based transient grating spectroscopy. In physics fundamental questions are under investigation regarding phase transitions, critical fluctuation in magnetic materials as well as nanometer scale ordering phenomena regarding charge, spin and orbital order in correlated materials. In order to probe these examples of nanometer scale dynamics, we have to extend transient grating methods towards shorter wavelengths, which FELs can deliver. Also for the technology of EUV lithography our understanding of dynamics induced by extreme ultraviolet (EUV) radiation at $13.5 \mathrm{~nm}$ wavelength are crucial for nanometer scale pattern transfer. Here the spatial resolution (structure size $32 \mathrm{~nm}$ ) and the line edge roughness is not only determined by the fundamental diffraction limit but by the diffusive processes within the photo-resist materials after EUV irradiation [15]. As the initial EUV photochemical reaction creates radicals and acids, which are the starting point of chemical amplification within the illuminated resist material during post-exposure baking [16-18], their initial spatial distribution, ultra fast dynamics as well as their collective dynamics and diffusion within the photoresist determine the achievable structure size and line edge roughness [15]. 

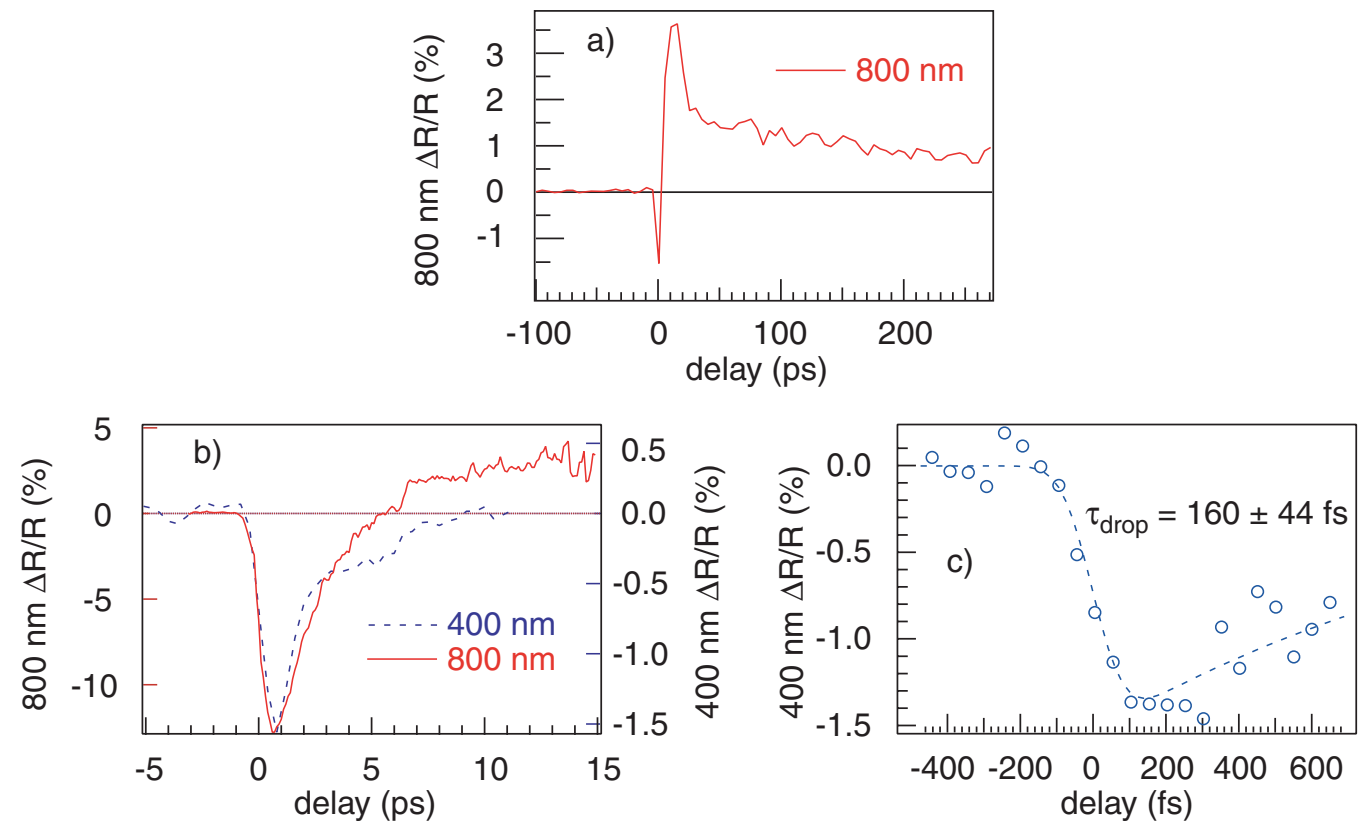

Fig. 2. Transient X-ray induced optical reflectivity $(\Delta R / R)$ - temporal characteristics: a) X-ray pulse induced dynamics: A rapid initial electronic response is followed by slower lattice dynamics on a picosecond timescale. b) Ultra fast electronic response after X-ray pump: The rapid drop of $\Delta \mathrm{R} / \mathrm{R}$ observed for $400 \mathrm{~nm}$ and $800 \mathrm{~nm}$ probe wavelength is followed by a recovery which depends on the GaAs band structure and the time scale of electronic relaxation. c) The width of the initial drop in optical reflectivity as determined fitting an exponentially decaying response function convoluted with a Gaussian (full width at half maximum of $160 \pm 44 \mathrm{fs}$ ) is limited by the pulse length of the optical laser pulse (120-150 fs). To eliminate temporal jitter from the accelerator the delay is corrected for the electron bunch arrival determined with electro optical sampling (EOS) [13].

With intense fs pulses from FLASH at $13.5 \mathrm{~nm}$ wavelength we now have a source at hand, which not only allows us to determine the ultra fast time scales of molecular processes, but also to determine the collective dynamics on a nanometer scale after EUV exposure in a transient grating experiment. In a first attempt, we have created a X-ray induced pattern on a GaAs wafer which we have probed by optical diffraction. To this end we inserted directly behind the FLASH undulator a fine gold mesh into the X-ray beam of $92 \mathrm{eV}$. As depicted schematically in Fig. 3 we focused the X-ray beam onto the GaAs sample surface through the refocussing optics of the FLASH beam line BL2 creating a $10 \mu \mathrm{m}$ focal spot. Within this focal plane a nanometer scale periodic pattern was formed modifying the optical constants of the GaAs surface as we have described before [1,2]. Shining optical laser light onto this surface yielded directly the optical diffraction pattern shown in Fig. 3 which was recorded detected through a CCD (charge coupled device) camera. Although in this first experiment we have not achieved temporal resolution by delaying the X-ray and optical pulses in an pump-probe arrangement, this result clearly demonstrates the potential to investigate collective dynamics such as radical diffusion and excited state-phonon interaction through X-ray induced transient grating experiments, where ideally the transient grating structure is created through the interference pattern of two FEL-beams and probed by a third beam. With the development of shorter wavelength FELs we will gain sufficient spatial resolution and chemical selectivity to study ultra fast dynamics from the atomic scale up to the nanometer scale of collective dynamics.

\section{Summary}

To harness the potential of X-ray free-electron lasers we have developed X-ray/optical crosscorrelation through X-ray induced transient optical reflectivity on GaAs. This allows to monitor 


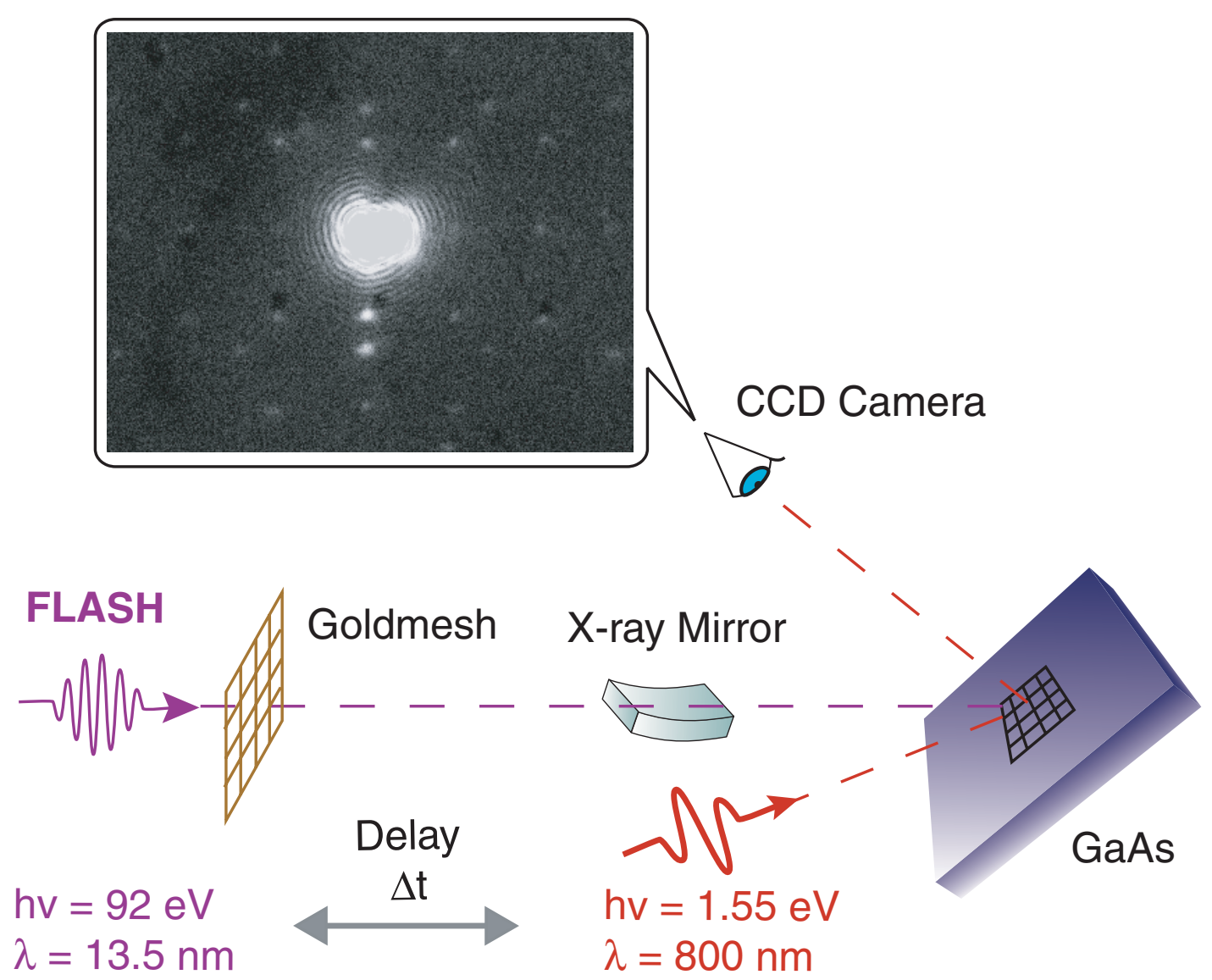

Fig. 3. Optical diffraction pattern observed through $800 \mathrm{~nm}$ optical laser light. The diffraction pattern has been created by imaging a gold mesh with FLASH femtosecond X-ray pulses onto a GaAs wafer used for X-ray induced transient optical reflectivity measurements.

at present and future free-electron lasers the temporal overlap at the experimental interaction point of the X-ray laser and synchronized optical lasers and to monitor their temporal jitter. As the underlying physical mechanism is the X-ray induced dynamics within solids, we explore the feasibility to determine with extreme ultraviolet (EUV) radiation $(13.5 \mathrm{~nm})$ in an X-ray induced transient grating experiment the ultra fast dynamics and the diffusion parameters within a solid to study the fundamental processes of EUV lithography.

We gratefully acknowledge support by the scientific and technical staff of the FLASH facility. This work was supported by the German Ministry of Education and Research (BMBF) Forschungsschwerpunkt FLASH through grants nos. 05 KS4GU1/8 and 05 KS4GU1/9, the Helmholtz Joint Research Centre "Physics with coherent radiation sources" and the Research Training Group/Graduiertenkolleg 1355 "Physics with new advanced coherent radiation sources" funded by the Deutsche Forschungsgemeinschaft.

\section{References}

1. C. Gahl, A. Azima, M. Beye, M. Deppe, K. Döbrich, U. Hasslinger, F. Hennies, A. Melnikov, M. Nagasono, A. Pietzsch, M. Wolf, W. Wurth, A. Föhlisch, Nat. Photon. 2, 165 (2008)

2. T. Maltezopoulos, S. Cunovic, M. Wieland, M. Beye, A. Azima, H. Redlin, M. Krikunova, R. Kalms, U. Frühling, F. Budzyn, W. Wurth, A. Föhlisch, M. Drescher, New J. Phys. 10, 033026 (2008) 
3. K.A. Nelson, M.D. Fayer, J. Chem. Phys. 72, 5202 (1980)

4. K.A. Nelson, R. Casalegno, R.J.D. Miller, M.D. Fayer, J. Chem. Phys. 77, 1144 (1982)

5. K.A. Nelson, R.J.D. Miller, D.R. Lutz, M.D. Fayer, J. Appl. Phys. 53, 1144 (1982)

6. E.J. Brown, Q.G. Zhang, M. Dantus, J. Chem. Phys. 110, 5772 (1999)

7. A. Pietzsch, A. Föhlisch, M. Beye, M. Deppe, F. Hennies, M. Nagasono, E. Suljoti, W. Wurth, C. Gahl, K. Döbrich, A. Melnikov, New J. Phys. 10, 033004 (2008)

8. S. Düsterer, P. Radcliffe, G. Geloni, U. Jastrow, M. Kuhlmann, E. Plönjes, K. Tiedtke, R. Treusch, J. Feldhaus, P. Nicolosi, L. Poletto, P. Yeates, H. Luna, J.T. Costello, P. Orr, D. Cubaynes, M. Meyer, Opt. Lett. 31, 1750 (2006)

9. P. Radcliffe, S. Düsterer, A. Azima, W.B. Li, E. Plönjes, H. Redlin, J. Feldhaus, P. Nicolosi, L. Poletto, J. Dardis, J.P. Gutierrez, P. Hough, K.D. Kavanagh, E.T. Kennedy, H. Luna, P. Yeates, J.T. Costello, A. Delyseries, C.L.S. Lewis, D. Glijer, D. Cubaynes, M. Meyer, Nucl. Instrum. Meth. Phys. Res. A 583, 516 (2007)

10. E.S. Toma, H.G. Muller, P.M. Paul, P. Breger, M. Cheret, P. Agostini, C. Le Blanc, G. Mullot, G. Cheriaux, Phys. Rev. A 62, 061801 (2000)

11. M. Meyer, D. Cubaynes, P. O'Keeffe, H. Luna, P. Yeates, E.T. Kennedy, J.T. Costello, P. Orr, R. Taïeb, A. Maquet, S. Düsterer, P. Radcliffe, H. Redlin, A. Azima, E. Plönjes, J. Feldhaus, Phys. Rev. A 74, 011401(R) (2006)

12. A. Cavalleri, et al., Phys. Rev. B 63, 193306 (2001)

13. A.L. Cavalieri, et al., Phys. Rev. Lett. 94, 114801 (2005)

14. J.-J. Yeh, I. Lindau, At. Data Nucl. Data Tables 32, 1 (1985)

15. A. Saeki, T. Kozawa, S. Tagawa, H.B. Cao, Nanotechnology 17, 1543 (2006)

16. H. Ito, C.G. Willson, Polym. Eng. Sci. 23, 1012 (1983)

17. E.K. Lin, et al., Science 297, 372 (2002)

18. W.D. Hinsberg, F.A. Houle, M.I. Sanchez, G.M. Wallraff, IBM J. Res. Dev. 45, 667 (2001) 